\title{
Amperometric Determination of Ascorbic Acid on an Au Electrode Modified by a Composite Film of Poly(3,4-ethylenedioxythiophene) and Superconductive Carbon Black
}

\author{
Xinfei Zhou,* Kunyun He,* Yu WAng, ${ }^{*}$ Haitao Zheng, ${ }^{* \dagger}$ and Shin-ichiro SuYE** \\ *School of Environmental and Chemical Engineering, Tianjin Polytechnic University, Tianjin 300387, \\ P. R. China \\ **Department of Applied Chemistry and Biotechnology, Graduate School of Engineering, University of Fukui, \\ 3-9-1 Bunkyo, Fukui 910-8507, Japan
}

\begin{abstract}
A new composite film-modified electrode was prepared by the electropolymerisation of poly(3,4-ethylenedioxythiophene) (PEDOT) and superconductive carbon black (SCB) on a gold electrode. The PEDOT-SCB/Au electrode exhibited excellent ability towards the electrocatalytic oxidation of ascorbic acid, in terms of a $480 \mathrm{mV}$ shift of the oxidation potential in the negative direction, and a dramatically enhanced oxidation current. Under the optimum conditions, the amperometric detection of ascorbic acid provided a wide linear detection range from $1.0 \times 10^{-7}$ to $8.0 \times 10^{-4} \mathrm{M}$, and a detection limit of $5.0 \times 10^{-8} \mathrm{M}(S / N=2)$ as well as good reproducibility and stability.
\end{abstract}

Keywords Ascorbic acid, poly(3,4-ethylenedioxythiophene), superconductive carbon black, cyclic voltammetry, chronoamperometry

(Received August 18, 2014; Accepted February 3, 2015; Published May 10, 2015)

\section{Introduction}

Ascorbic acid (AA) is a powerful natural antioxidant and a vital component in the human diet, and is present in both the animal and plant kingdoms. ${ }^{1,2}$ Its precursors and metabolites include one of the most important biological compounds, participating in several important biological processes, such as the regulation of cell division, flowering phase induction and adjustment, stomatal closure, growth and development. ${ }^{3,4}$ In addition, AA has been used for preventing and treating lots of diseases, including the common cold, mental illness, infertility, cancer, AIDS and so on. ${ }^{5,6}$ It is also widely used in both foods and drinks as a powerful antioxidant agent. ${ }^{7,8}$ In view of these functionalities, the determination of AA has received great attention in biomedical engineering, the food and agricultural industries as well as clinical applications. ${ }^{9-11}$ However, AA always coexists with dopamine (DA) and uric acid (UA) in physiological samples, ${ }^{12,13}$ and many conventional methods have been introduced to detect it, such as chromatography, ${ }^{14}$ UV-visible spectrometry, ${ }^{15}$ fluorimetry ${ }^{16}$ and high-performance liquid chromatography. ${ }^{17}$ Since, all of them are involved in several disadvantages, such as time-consuming, multi-step procedures and interference resulting from other chemicals, ${ }^{18,19}$ it is urgent to develop fast, sensitive and inexpensive methods to detect AA from its coexistences.

Electrochemical sensors have received great interest for the determination of AA because of their high sensitivity, simple

$\dagger$ To whom correspondence should be addressed.

E-mail: zhenght999@hotmail.com operation, easy miniaturization and low cost. ${ }^{20,21}$ The electrochemical determination of AA is generally based on its reducing properties, ${ }^{22}$ but it is difficult to determine AA by its direct oxidation, because the oxidation potentials of AA, DA and UA are very similar on bare electrodes. ${ }^{23-25}$ On the other hand, the direct oxidation of AA on bare electrodes is irreversible, which not only requires a high overpotential, but also suffers from a pronounced fouling effect on the surface, resulting in poor sensitivity and selectivity. ${ }^{26}$ To overcome the above problems, a number of modified electrodes have been developed for the selective determination of AA, DA and UA, such as conducting polymers, ${ }^{16,26,27}$ carbon nanotubes, ${ }^{18,28,29}$ metal nanoparticles, ${ }^{21,30-32}$ nanofibers ${ }^{33}$ and graphite. ${ }^{34}$ Among these methods, a conducting polymers modified electrode exhibited excellent capability in the determination of AA for their high electrocatalytic ability, good stability and broad potential windows. Furthermore, they can provide a suitable microenvironment for the immobilization of biomolecules, and act as mediators. ${ }^{11,35,36}$

Poly(3,4-ethylenedioxythiophene) (PEDOT), due to its high conductivity, good stability in both aqueous and nonaqueous forms, low interface impedance, reduced band-gap and environment-friendly features has received great interest in recent years. ${ }^{37-40}$ Besides the above advantages, the super biocompatibility and low Young's modulus also results in its wide application in preparation the modified electrodes and electrochemical sensing systems. ${ }^{41}$

In order to improve the properties of PEDOT film, some other materials, such as carbon nanotubes and graphite, were also used as conducting fillers. ${ }^{42-44}$ The PEDOT matrix incorporated with these materials exhibited superior selectivity and sensitivity 
compared with the polymer alone. In our previous work, hydroquinone and catechol were determined on a PEDOT modified electrode, which showed a higher sensitivity and selectivity. ${ }^{45}$ In this work, superconductive carbon black (SCB), owing to its superior conductivity, large specific surface area, high stability and better integration, was selected as a functional incorporation component to prepare a PEDOT-SCB composite film modified gold electrode. ${ }^{46-48}$ To the best of our knowledge, there has been no report on the fabrication and application of a PEDOT-SCB modified Au electrode for the electrocatalytic oxidation and determination of AA. Herein, the electrochemical behavior of AA on a PEDOT-SCB modified Au electrode was thoroughly investigated, and a sensitive electrochemical sensor for the determination of AA was also developed.

\section{Experimental}

\section{Reagents}

A 3,4-ethylenedioxythiophene (EDOT) was obtained from XZL Bio-Technology Co., Ltd. Lithium perchlorate $\left(\mathrm{LiClO}_{4}\right)$ was purchased from Guangfu Fine Chemical Research Institute. Acetonitrile and ascorbic acid (AA) were taken from Tianjin Kemiou Chemical Reagent Co., Ltd. SCB was obtained from Beijing Adana Holdings Technology Development Co., Ltd. Dopamine hydrochloride (DA) was purchased from Aladdin Co., Ltd. All other chemicals were of analytical grade, and were used without further purification. All solutions were prepared with double-distilled water. All experiments were carried out at ambient temperature, and highly pure nitrogen was used for deaeration.

\section{Instrumentation}

The electrochemical measurements were carried out on an LK2006A electrochemical workstation (Tianjin Lanlike Chemical and Electron High Technology Co., Ltd.). We employed a conventional three-electrode system comprising a platinum wire as the counter electrode, an $\mathrm{Ag} / \mathrm{AgCl}$ (sat'd $\mathrm{KCl}$ ) electrode as the reference electrode and an $\mathrm{Au}$ electrode or a modified Au electrode (Aida Instruments Corp., Tianjin, China, $1.6 \mathrm{~mm}$ in diameter) as the working electrode. A scanning electron microscope (SEM) (Quanta 200, FEI) and fieldemission scanning electron microscope (FE-SEM) (S-4800, HITACHI) were used to characterize the surface of a modified electrode.

\section{Preparation of a PEDOT-SCB modified electrode}

The SCB sample was firstly treated in a mixed solution of strong acids to obtain a fully protonated SCB. Briefly, $1.0 \mathrm{~g}$ of SCB was dispersed in $100 \mathrm{~mL}$ of a $3: 1$ concentrated $\mathrm{H}_{2} \mathrm{SO}_{4}$ (98\%) and $\mathrm{HNO}_{3}$ solution (65\%). After sonication for $5 \mathrm{~h}$, the mixture was left under stirring overnight, and then filtered. The solid was washed with double-distilled water until the $\mathrm{pH}$ was neutral. Finally, the SCB was collected and dried in a vacuum at $80^{\circ} \mathrm{C}$ for $2 \mathrm{~h}$.

Before any modification, the bare Au electrodes were finely polished by a 1.0 and $0.05 \mu \mathrm{m}$ aluminum slurry, then rinsed thoroughly with double-distilled water, followed by an ultrasonic treatment for $5 \mathrm{~min}$. Finally, they were scanned over the potential range of 0.0 to $+1.7 \mathrm{~V}(v s . \mathrm{Ag} / \mathrm{AgCl})$ in a $0.5 \mathrm{M} \mathrm{H}_{2} \mathrm{SO}_{4}$ solution about 30 times until the same voltammograms were obtained. A PEDOT-SCB modified electrode was prepared by one-step electrochemical polymerization in a $0.1 \mathrm{M}$ EDOT acetonitrile solution containing $0.1 \mathrm{M} \mathrm{LiClO}_{4}$ and $100 \mathrm{mg} \mathrm{L}^{-1}$ $\mathrm{SCB}$ at a positive potential of $+1.3 \mathrm{~V}$ for an appropriate time.
After electropolymerization, the PEDOT-SCB modified electrode was washed with double-distilled water and dried by $\mathrm{N}_{2}$.

\section{Electrochemical measurement}

A three-electrode electrochemical system was used in cyclic voltammetry and amperometric experiments. The electrochemical behaviors of AA on different electrodes were investigated by cyclic voltammetry between -0.4 and $+0.7 \mathrm{~V}$ in a $0.1 \mathrm{M}$ phosphate buffer solution (PBS). The applied potential was then set at $+0.02 \mathrm{~V}$ for an amperometric determination.

\section{Results and Discussion}

\section{Characterization of the PEDOT-SCB modified Au electrode}

Firstly, the surface morphology of the PEDOT-SCB modified $\mathrm{Au}$ electrode and the PEDOT-SCB film were characterized by SEM and FE-SEM, respectively. The surface of the bare Au electrode was almost smooth, as shown in Fig. 1A, and the electrode surface was covered with a pyknotic polymer film obviously, after electropolymerization of the PEDOT (shown in Fig. 1B), which indicated that the PEDOT has been polymerized on the Au surface. ${ }^{45}$ A pore rules structure of SCB was found when the $\mathrm{Au}$ electrode was modified by a composite film of both PEDOT and SCB (shown in Figs. 1C and 1D), and the SCB distributed homogeneously in the PDEOT film, which was proved by section images of the PEDOT-SCB film, shown in Figs. 1E and 1F. This suggested that a PEDOT-SCB composite film had been successfully fabricated on the electrode surface by the present method.

The electrochemical properties of the PEDOT-SCB modified electrode was further characterized by electrochemical impedance spectroscopy (EIS), which was performed in a $2.5 \mathrm{mM}\left[\mathrm{Fe}(\mathrm{CN})_{6}\right]^{3-} /\left[\mathrm{Fe}(\mathrm{CN})_{6}\right]^{4-}$ solution. The data was shown in Fig. 2. In Nyquist plots of EIS, the semicircle part at higher frequencies corresponds to an electron-transfer limited process, and the linear part at lower frequencies corresponds to the diffusion-controlled process. For a bare $\mathrm{Au}$ electrode, the electron-transfer resistance $\left(R_{\mathrm{et}}\right)$ was $2800 \Omega$, and after modifying the PEDOT and PEDOT-SCB film, the $R_{\mathrm{et}}$ value decreased to 1635 and $910 \Omega$, respectively. These results indicated that the Au electrode was successfully modified by the PEDOT or PEDOT-SCB film. On the other hand, it is also suggested that the incorporated SCB in the PEDOT matrix is helpful to improve the conductivity of the PEDOT film.

\section{Electrochemical behavior of AA on a PEDOT-SCB modified} electrode

The electrochemical behavior of AA on the modified electrode was investigated by cyclic voltammetry. Figure 3 displays cyclic voltammograms of bare $\mathrm{Au}$ (a), PEDOT/Au (b) and PEDOT-SCB/Au (c) electrode in $0.1 \mathrm{M}$ PBS ( $\mathrm{pH} 7$ ) containing $10.0 \mathrm{mM}$ AA. As shown in Fig. 3, there is a broad oxidation peak at around $+0.5 \mathrm{~V}$ for the bare $\mathrm{Au}$ electrode (curve a). In contrast, a well-defined oxidization peak was observed at $+0.02 \mathrm{~V}$ on a PEDOT (curve b) or PEDOT-SCB modified Au electrode (curve c), and the oxidation potential decreased by $480 \mathrm{mV}$ approximately. The negative potential shift of AA on a PEDOT or PEDOT-SCB modified electrode should be attributed to an electrostatic attraction between the negatively charged ascorbate anion and the oxidation-form of the PEDOT film, which led to a pre-concentration of the analyte on the electrode surface. ${ }^{40,49}$ On the other hand, SCB enhanced the electrode surface area as well as the electroactivity and conductivity of the 

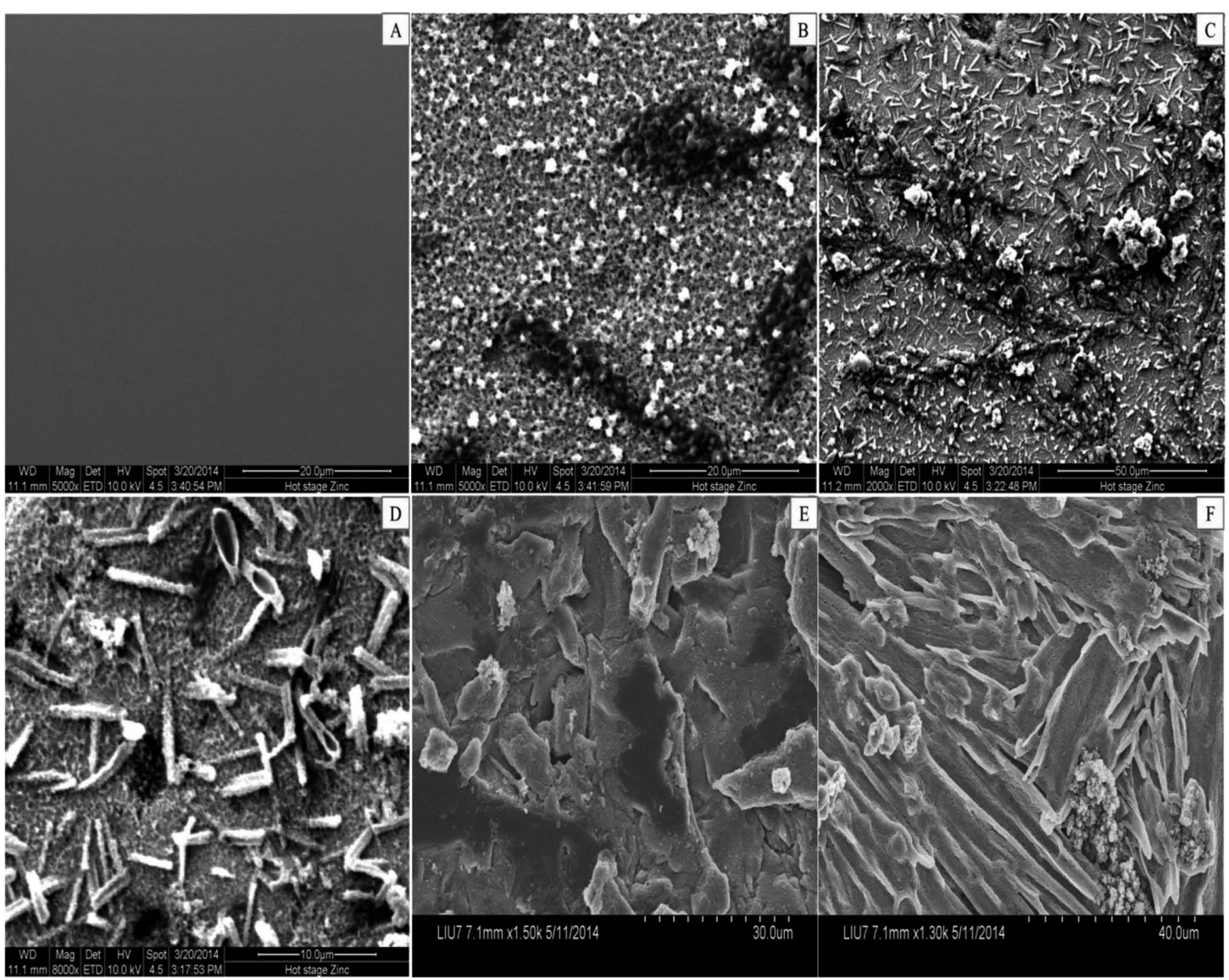

Fig. 1 SEM images of bare Au (A), PEDOT/Au (B) and PEDOT-SCB/Au electrode (C and D). FE-SEM section images of PEDOT (E) and PEDOT-SCB (F) film.

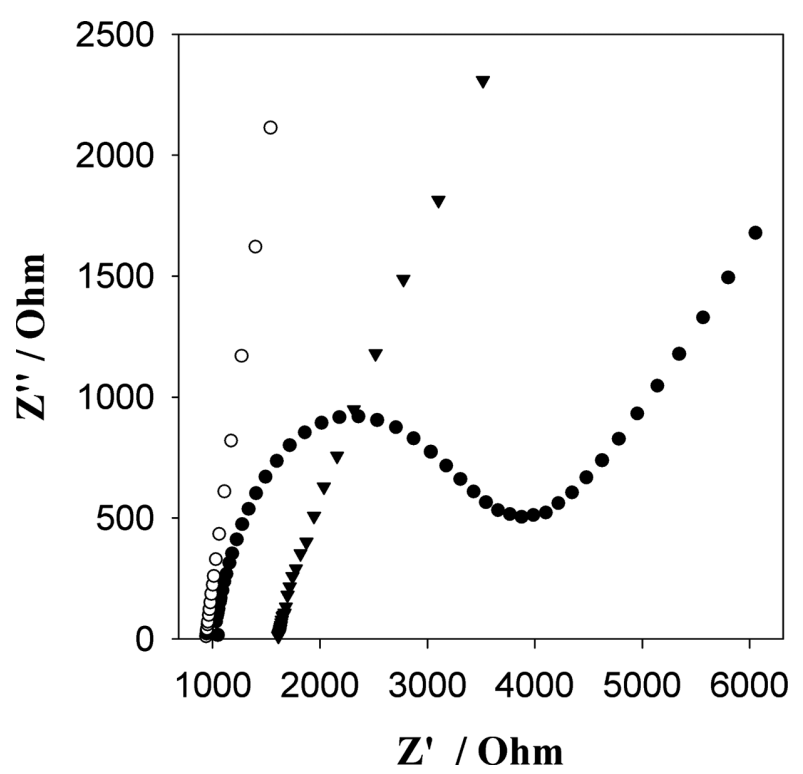

Fig. 2 EIS of bare Au $(\bullet)$, PEDOT/Au ( $\mathbf{\nabla})$ and PEDOT-SCB/Au (O) electrode in a $2.5 \mathrm{mM}\left[\mathrm{Fe}(\mathrm{CN})_{6}\right]^{3-/}\left[\mathrm{Fe}(\mathrm{CN})_{6}\right]^{4-}$ solution.

composite film, which resulted in a larger oxidation peak current compared with an electrode modified with the polymer alone.

The effect of the scan rate $(v)$ on the anodic peak current of AA was studied at PEDOT-SCB modified Au by cyclic

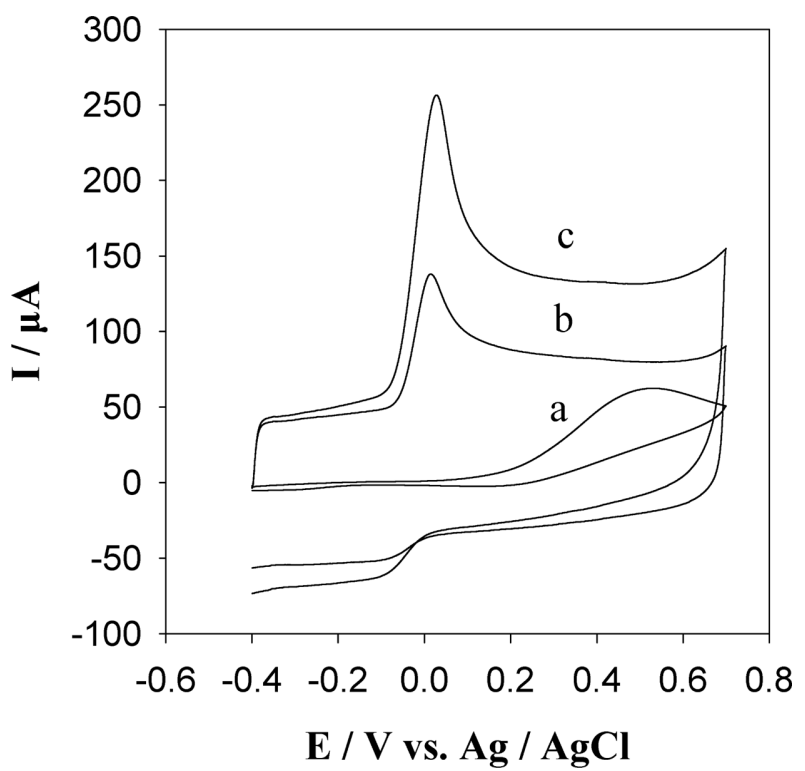

Fig. 3 Cyclic voltammograms of bare Au (a), PEDOT/Au (b) and PEDOT-SCB/Au (c) electrode in $0.1 \mathrm{M}$ PBS (pH 7) containing $10.0 \mathrm{mM}$ AA. Scan rate $0.1 \mathrm{~V} \mathrm{~s}^{-1}$.

voltammetry in the range of 0.01 to $0.1 \mathrm{~V} \mathrm{~s}^{-1}$, (shown in Fig. 4). It was clearly found that the anodic peak current increased with the scan rate (Fig. 4A), and varied linearly with the square root 

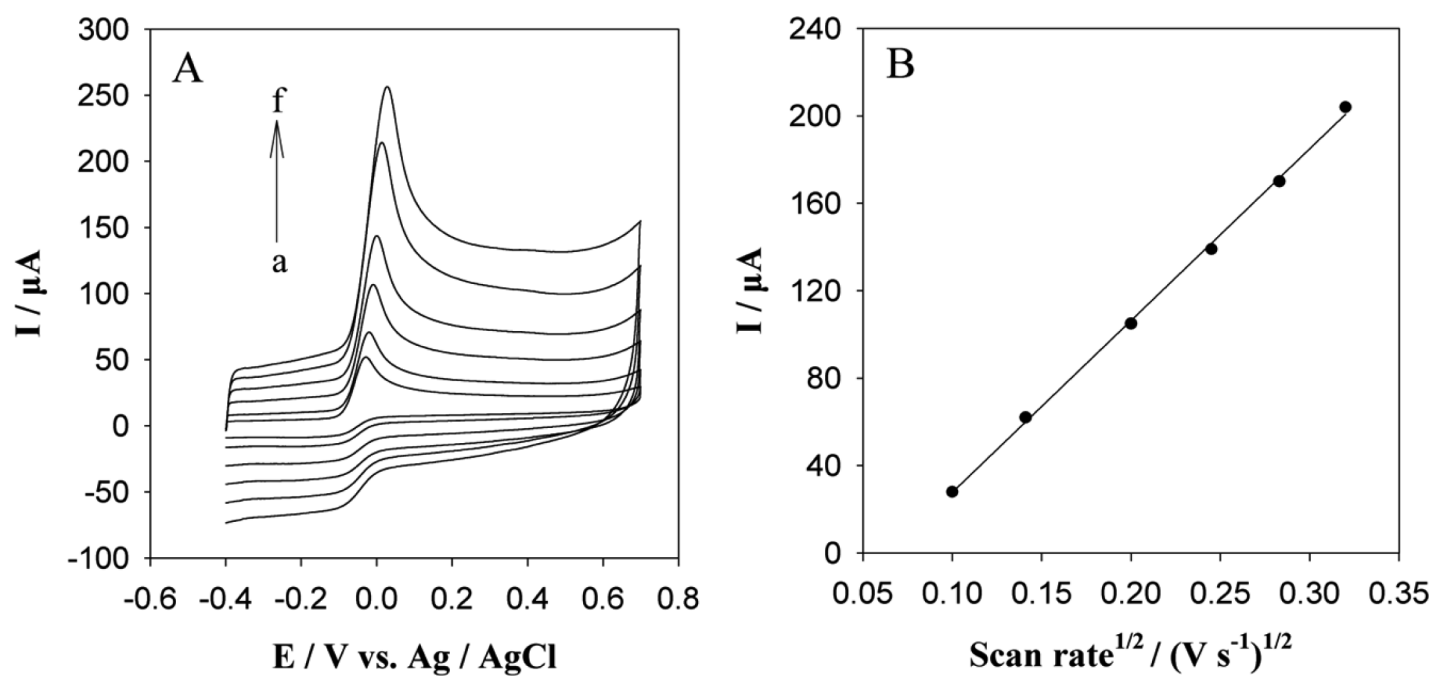

Fig. 4 (A) Cyclic voltammograms of PEDOT-SCB/Au in $0.1 \mathrm{M} \mathrm{PBS} \mathrm{(pH} \mathrm{7)} \mathrm{containing} 10.0 \mathrm{mM} \mathrm{AA}$ at scan rates of 0.01 (a), 0.02 (b), 0.04 (c), 0.06 (d), 0.08 (e) and 0.1 (f) $\mathrm{V} \mathrm{s}^{-1}$ from inner to outer; (B) Relationship between the square root of the scan rate and the anodic peak current.
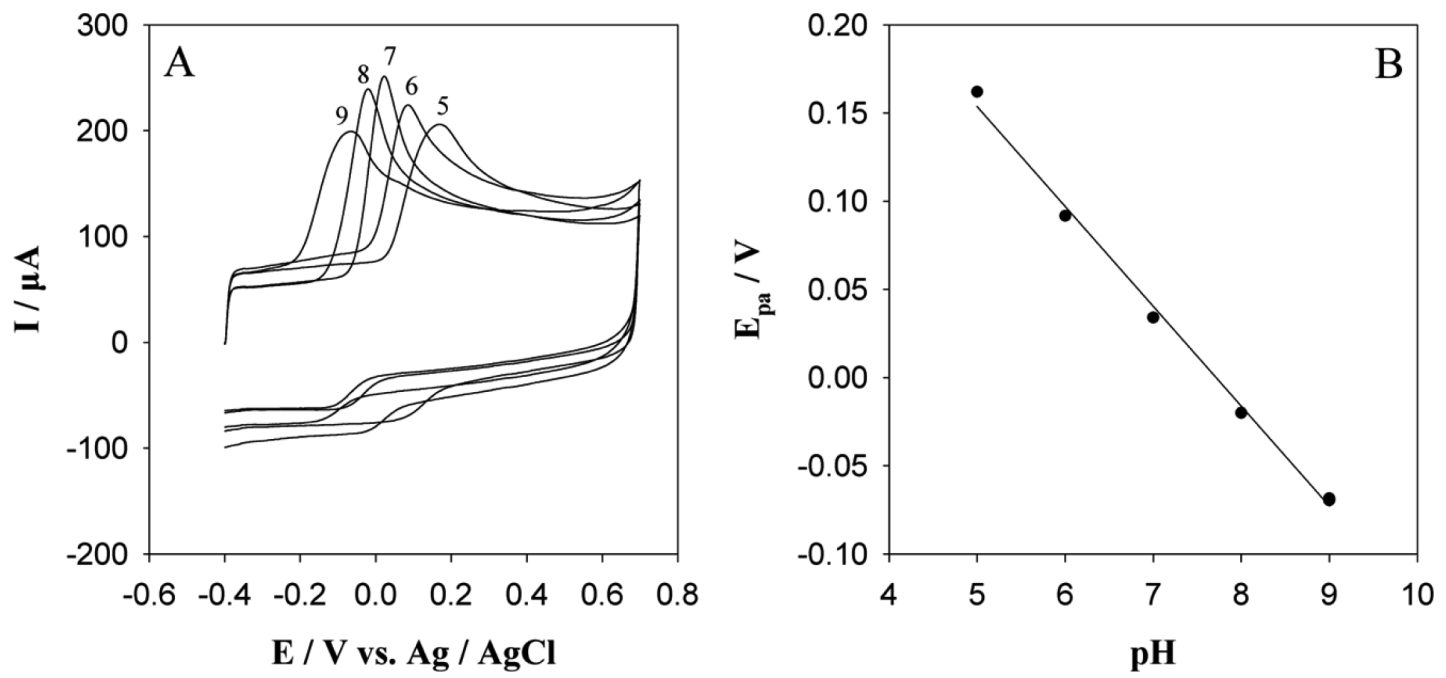

Fig. 5 A. Cyclic voltammograms of a PEDOT-SCB/Au electrode in $0.1 \mathrm{M}$ PBS containing $10.0 \mathrm{mM}$ AA with a scan rate of $0.1 \mathrm{~V} \mathrm{~s}^{-1}$ at a different $\mathrm{pH}$ values (from 5 to 9), B. Effect of the $\mathrm{pH}$ on the peak potentials of AA.

of the scan rate (Fig. 4B), with a linear-regression equation of $I_{\mathrm{pa}}=768.0 v^{1 / 2}-50.87\left(I_{\mathrm{pa}}: \mu \mathrm{A}, v^{1 / 2}:\left(\mathrm{V} \mathrm{s}^{-1}\right)^{1 / 2}\right)\left(R^{2}=0.998\right)$, which confirmed that the oxidation of AA on the surface of PEDOT-SCB/Au is a diffusion-controlled process.

\section{The effect of the $\mathrm{pH}$}

Because the $\mathrm{pH}$ value has a profound effect on the electrochemical behavior of AA, the oxidation was studied in a solution containing $10.0 \mathrm{mM}$ AA with different $\mathrm{pH}$ values on a PEDOT-SCB modified electrode. As shown in Fig. 5, the catalytic peak shifted to a more negative potential with an increase of the $\mathrm{pH}$ value (Fig. 5A). The relationship between the oxidation potential and the $\mathrm{pH}$ was nearly linear (Fig. 5B), with the linear-regression equation being of $E_{\mathrm{pa}}(\mathrm{V})=-0.057 \mathrm{pH}$ $+0.442\left(R^{2}=0.995\right)$. Its slope was very close to the Nernstian value of $-59 \mathrm{mV}$, which indicated that the oxidation of AA involved the same number of electrons and protons at $\mathrm{pH}$ values from 5 to 9. These conclusions are in accordance with the mechanism of the electrochemical redox process of AA.

\section{Amperometric determination of $A A$}

The chronoamperometry current response of a PEDOT-SCB modified $\mathrm{Au}$ electrode was investigated to concerning the successive addition of AA into 0.1 M PBS (pH 7) at an applied potential of $+0.02 \mathrm{~V}$. Figure $6 \mathrm{~A}$ shows the corresponding responses with increasing concentrations of $0.1,1.0,10.0$ and $100.0 \mu \mathrm{M}$. A subsequent addition of the substrate caused a remarkable increase in the anodic current, and the time required to reach a $95 \%$ steady state was within $10 \mathrm{~s}$. The fast response might have resulted from the unique structure of the PEDOT film and pore rules structure of SCB incorporated in the PEDOT matrix. On the other hand, both PEDOT and SCB have so a very high electron-transfer capability, which greatly improved the electrocatalytic response of AA. 

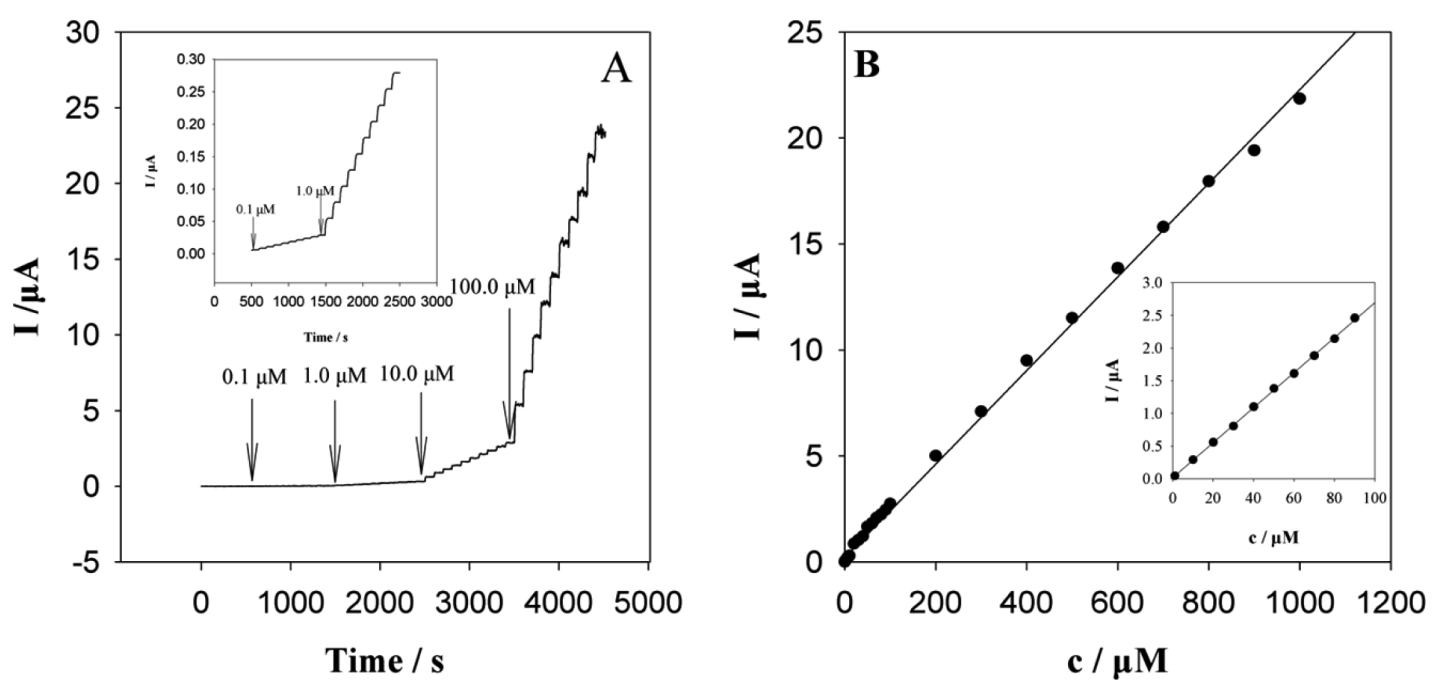

Fig. 6 (A) Typical current-time response curve of the PEDOT-SCB/Au electrode to the successive addition of AA at an applied potential of $+0.02 \mathrm{~V}(\mathrm{pH} 7)$. (B) Relationship between the substrate concentrations and the current response. The inset shows the curve for low AA concentrations.

Table 1 Comparison of the analytical performances at various electrodes reported earlier for AA determination

\begin{tabular}{|c|c|c|c|c|c|c|}
\hline Modified electrode & $\mathrm{pH}$ & $E_{\mathrm{pa}}$ & Linear range $/ \mu \mathrm{M}$ & Detection limit/ $\mu \mathrm{M}$ & $S / N$ & Ref. \\
\hline La-MWCNTsa/GCE & 6.0 & $80 \mathrm{mV}$ vs. SCE & $0.4-710$ & 0.14 & 3 & 28 \\
\hline Poly-ACBK $/$ GCE & 4.0 & $120 \mathrm{mV} v s . \mathrm{SCE}$ & $50-1000$ & 10.0 & 3 & 1 \\
\hline SWCNTs/GCE & 4.5 & $240 \mathrm{mV}$ vs. SCE & $15-800$ & 6.68 & - & 24 \\
\hline GDSPc/CPE & 7.0 & $380 \mathrm{mV}$ vs. SCE & $150-800$ & 3.375 & 3 & 31 \\
\hline PEDOT/Au & 7.0 & $-94 \mathrm{mV} v s . \mathrm{SCE}$ & $5-300$ & 2.5 & - & 20 \\
\hline e-FGPE ${ }^{d}$ & 7.0 & $-100 \mathrm{mV}$ vs. $\mathrm{Ag} / \mathrm{AgCl}$ & $20-400$ & 2.0 & 3 & 13 \\
\hline $\mathrm{PAF} / \mathrm{GCE}$ & 7.0 & $200 \mathrm{mV}$ vs. $\mathrm{Ag} / \mathrm{AgCl}$ & $3-200$ & 1.5 & - & 26 \\
\hline PEDOT-MWCNTs/GCE & 7.0 & $100 \mathrm{mV}$ vs. $\mathrm{Ag} / \mathrm{AgCl}$ & $100-2000$ & 100 & 2 & 44 \\
\hline PEDOT-SCB/Au & 7.0 & $20 \mathrm{mV}$ vs. $\mathrm{Ag} / \mathrm{AgCl}$ & $0.1-800$ & 0.05 & 2 & This works \\
\hline
\end{tabular}

a. Lathanum-multiwalled carbon nanotube.

b. Poly (acid chrome blue K).

c. Gold decorated $\mathrm{SiO}_{2} @$ polyaniline core-shell microspheres.

d. Exfoliated flexible graphite paper electrode.

e. Ploy (acriflavine).

The relationship between the current response and the concentration of AA is shown in Fig. 6B. The inset shows the curve for low AA concentrations. It is clearly found that the current response is linearly proportional to its concentration in the range of $1.0 \times 10^{-7}$ to $8.0 \times 10^{-4} \mathrm{M}$, and that the regression equation is $I_{\mathrm{h}}=0.022 c+0.208\left(I_{\mathrm{h}}: \mu \mathrm{A}, c: \mu \mathrm{M}\right)\left(R^{2}=0.998\right)$. This linear range covers the natural range of human blood (34 $80 \mu \mathrm{M}$ ), and a lower detection limit of $5.0 \times 10^{-8} \mathrm{M}$ was obtained with a signal-to-noise ratio $(S / N)$ of 2 .

The results obtained in the present work were also compared with that of other researchers. A summary of the modified electrodes developed for the determination of AA, their oxidation potentials, detection limits and linear ranges are listed in Table 1. It shown that the oxidation potential of AA in the present work decreased greatly, and that the detection limit is lower than that in works published previously. This should be attributed to the unique structure of PEDOT-SCB film, which not only enhanced the electron transfer, but also greatly improved the electrocatalytic ability toward the AA.

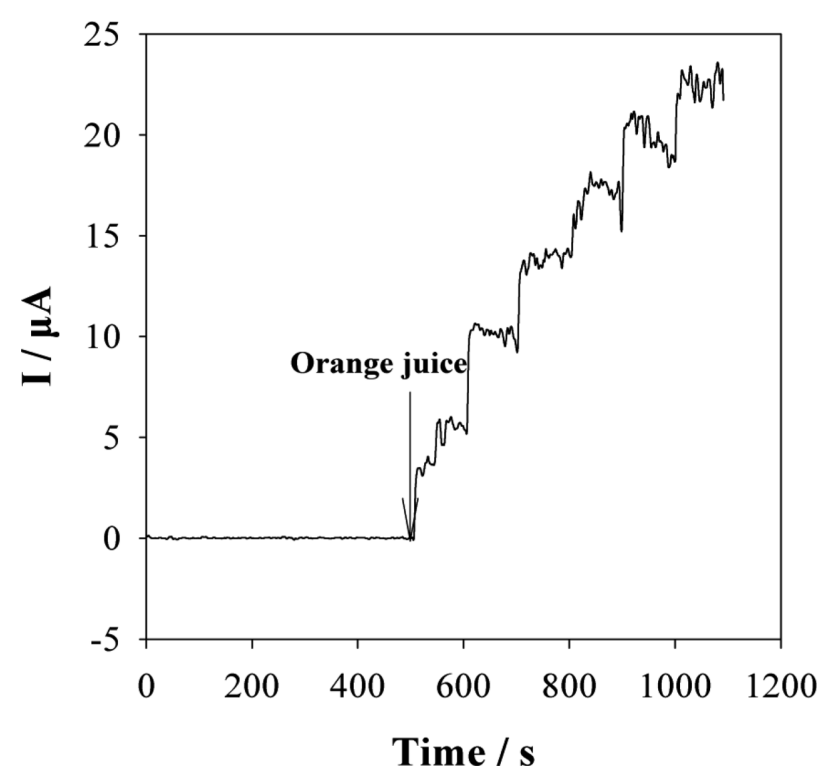

Fig. 7 Amperometric current-time curve for an AA estimation in orange juice at a PEDOT-SCB/Au electrode. Each step represents a $1.0 \mathrm{~mL}$ addition of natural orange juice. 
Application for real sample analysis

To check the practicability of the PEDOT-SCB modified electrode, the AA content in orange juice has been established directly with this electrode by an amperometric method. Figure 7 shows a typical amperometric current-time response recorded for the oxidation of AA in orange juice for sequential additions of $1.0 \mathrm{~mL}$ of the as-bought orange juice, without any dilution, to a $9 \mathrm{~mL} 0.1 \mathrm{M}$ PBS solution ( $\mathrm{pH} 7$ ) at an applied potential of $+0.02 \mathrm{~V}$ with a constant stirring rate of $300 \mathrm{rpm}$. A fast current response can be seen when $1.0 \mathrm{~mL}$ of orange juice was added into the PBS solution, an average AA concentration of $38 \mathrm{mg} / 100 \mathrm{~mL}$ of juice was obtained over ten measurements with a small relative standard deviation of $1.97 \%$, which means that the PEDOT-SCB modified electrode can be used for orange juice analysis. The values were monitored by a PEDOT-SCB sensor and HPLC, as shown in Table 2.

\section{Interference test}

Because dopamine (DA), uric acid (UA) and NADH are among the common interferences, whose oxidization potentials overlap with AA on bare electrodes, their effects were examined. Curve $\mathrm{f}$ in Fig. 8A shows a cyclic voltammogram of a solution including $2.5 \mathrm{mM}$ AA, $2.5 \mathrm{mM} \mathrm{DA}, 2.5 \mathrm{mM}$ UA and $5.0 \mathrm{mM}$ $\mathrm{NADH}$, measured at a bare Au electrode. Only one broad peak appeared for these analytes. Electrode fouling occurred due to the adsorption of an oxidized product, which caused only a single peak. ${ }^{50}$ However, AA, DA, UA and NADH were electrochemically oxidized at different potentials, as shown in curves a, b, c, and d, which suggest that the PEDOT-SCB film

Table 2 Determination of the AA concentration using both the PEDOT-SCB sensor and HPLC for orange juice

\begin{tabular}{llll}
\hline & $\begin{array}{c}\text { Concentration } \\
\text { specified on tag }\end{array}$ & $\begin{array}{c}\text { AA sensor of } \\
\text { PEDOT-SCB }\end{array}$ & HPLC \\
\hline Orange juice & $40 \mathrm{mg} / 100 \mathrm{mg}$ & $\begin{array}{l}38 \mathrm{mg} / 100 \mathrm{~mL} \\
(\text { Error }=1.97 \%)\end{array}$ & $39.1 \mathrm{mg} / 100 \mathrm{~mL}$ \\
\hline
\end{tabular}



had excellent ability concerning electrochemical catalyzation for each component. A cyclic voltammogram of the mixture of AA, DA, UA and NADH (curve e) showed separated oxidation peaks for AA, DA, and UA, but the oxidation peak of NADH was covered by the broad peak of UA, which indicated that the oxidation of AA can not be disturbed by DA, UA and NADH on a PEDOT-SCB modified electrode.

Figure $8 \mathrm{~B}$ shows the amperometric responses of AA, DA, UA and NADH on a PEDOT-SCB modified Au electrode at an applied potential of $+0.02 \mathrm{~V}$. There is a fast current response after the addition of $0.1 \mathrm{mM} \mathrm{AA}$, and no current response appeared after the injection of $0.1 \mathrm{mM}$ DA, UA and NADH, but a fast current response appeared again after the addition of $0.1 \mathrm{mM}$ AA. This means that AA can be amperometrically determined on a PEDOT-SCB modified Au electrode in the

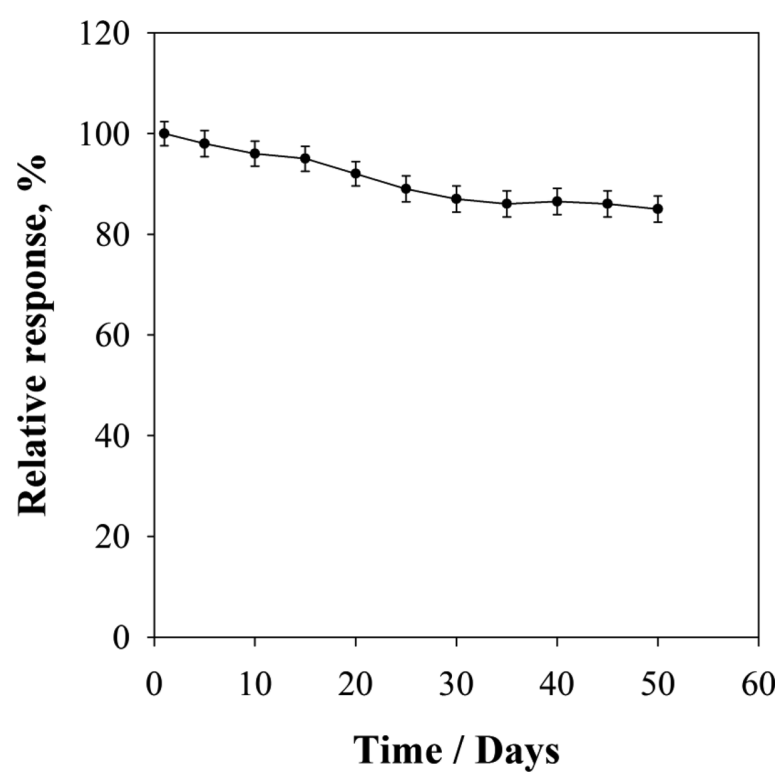

Fig. 9 Long-term storage stability of the AA sensor.



Fig. 8 (A) Cyclic voltammograms of $2.5 \mathrm{mM} \mathrm{AA}$ (a), $2.5 \mathrm{mM}$ DA (b), $2.5 \mathrm{mM}$ UA (c) and $5.0 \mathrm{mM}$ NADH (d) on PEDOT-SCB modified electrode, and a mixture of the above components on a PEDOTSCB modified (e) and bare electrode (f) with a scan rate of $0.1 \mathrm{~V} \mathrm{~s}^{-1}$. (B) Current-time response of AA, DA, UA and NADH on PEDOT-SCB/Au electrode at an applied potential of $+0.02 \mathrm{~V}$. 
presence of DA, UA and NADH.

\section{Reproducibility and stability of the sensor}

The reproducibility and storage stability are very important for a sensing system. In this work, a test of the reproducibility was performed on four PEDOT-SCB modified electrodes prepared under the same conditions, and the relative standard deviation (RSD) was calculated to be $2.8 \%$, which revealed a good reproducibility. The storage stability of the PEDOT-SCB modified electrode was also investigated, with $0.1 \mathrm{~mL}$ of $10 \mathrm{mM}$ AA was added into $10 \mathrm{~mL}$ of $0.1 \mathrm{M}$ PBS solution. The modified electrode gave about $85 \%$ of its initial value after 50 days of storage in $0.1 \mathrm{M}$ PBS under $4^{\circ} \mathrm{C}$, which was measured at intervals of 5 days (Fig. 9). This excellent stability behavior was most probably due to the stability effect of PEDOT and SCB in both aqueous and non-aqueous states, which made the PEDOT-SCB film to have good chemical inertness. Thus, this electrode showed excellent stability.

\section{Conclusions}

In this study, a novel modified electrode was successfully fabricated by the electropolymerization of a composite film of PEDOT and SCB on the surface of a Au electrode, which was proved by SEM, FE-SEM and electrochemical methods. The modified electrode exhibited a fantabulous electrocatalytic ability toward AA, which not only decreased the oxidation potential of AA dramatically, but also enhanced the peak current greatly. A wide linear detection range of $1.0 \times 10^{-7}$ to $8.0 \times$ $10^{-4} \mathrm{M}$ and a lower detection limit of $5.0 \times 10^{-8} \mathrm{M}(S / N=2)$ were achieved under the optimum conditions $(\mathrm{pH} 7)$. On the other hand, the sensor also exhibited excellent reproducibility and stability, which suggested that the PEDOT-SCB modified $\mathrm{Au}$ electrode could be used as a promising sensing platform for the determination of AA.

\section{Acknowledgements}

The research was supported by the Scientific Research Foundation for the Returned Overseas Chinese Scholars (ROCS), Ministry of Education, P. R. China and Natural Science Foundation of Tianjin (No. 06YFJMJC15000), P. R. China.

\section{References}

1. R. Zhang, G. D. Jin, D. Chen, and X. Y. Hu, Sens. Actuators, B, 2009, 138, 174 .

2. C. Koçak and Z. Dursun, J. Electroanal. Chem., 2013, 694, 94.

3. Z. Chen and D. R. Gallie, Plant Cell, 2004, 16, 1143.

4. Y. Wen, J. Xu, M. Liu, D. Li, L. Lu, R. Yue, and H. He, J. Electroanal. Chem., 2012, 674, 71.

5. X. Zheng, X. Zhou, X. Ji, R. Lin, and W. Lin, Sens. Actuators, B, 2013, 178, 359.

6. H. Beitollahi and S. Mohammadi, Chin. J. Catal., 2013, 34, 1098.

7. X. Zhang, Y. Cao, S. Yu, F. Yang, and P. Xi, Biosens. Bioelectron., 2013, 44, 183.

8. X. Wang, P. Wu, X. Hou, and Y. Lv, Analyst, 2013, 138, 229.

9. D. Micić, B. Šljukić, Z. Zujovic, J. Travas-Sejdic, and G. Ćirić-Marjanović, Electrochim. Acta, 2014, 120, 147.
10. X. Zhang, S. Yu, W. He, H. Uyama, Q. Xie, L. Zhang, and F. Yang, Biosens. Bioelectron., 2014, 55, 446.

11. M. Liu, Y. Wen, D. Li, R. Yue, J. Xu, and H. He, Sens. Actuators, B, 2011, 159, 277.

12. H. D. Jirimali, R. K. Nagarale, D. Saravanakumar, J. M. Lee, and W. Shin, Carbohydr. Polym., 2013, 92, 641.

13. W. Cai, T. Lai, H. Du, and J. Ye, Sens. Actuators, B, 2014, 193, 492.

14. M. Mazloum-Ardakani, F. Habibollahi, H. R. Zare, H. Naeimi, and M. Nejati, J. Appl. Electrochem., 2009, 39, 1117.

15. M. Nicolleta, B. Semaghiul, D. Simona, and C. Petre, Acta Chim. Slov., 2004, 51, 169.

16. R. Thangamuthu, S. M. Senthil Kumar, and K. Chandrasekara Pillai, Sens. Actuators, B, 2007, 120, 745.

17. R. Leubolt and H. Klein, J. Chromatogr. A, 1993, 640, 271.

18. A. Kutluay and M. Aslanoglu, Sens. Actuators, B, 2013, 185, 398.

19. Y. P. Dong, L. Huang, J. Zhang, X. F. Chu, and Q. F. Zhang, Electrochim. Acta, 2012, 74, 189.

20. F. Sekli-Belaidi, P. Temple-Boyer, and P. Gros, J. Electroanal. Chem., 2010, 647, 159.

21. T. Madrakian, E. Haghshenas, and A. Afkhami, Sens. Actuators, B, 2014, 193, 451.

22. J. B. Raoof, R. Ojani, and A. Kiani, J. Electroanal. Chem., 2001, 515, 45.

23. V. S. Vasantha and S. M. Chen, Electrochim. Acta, 2005, $51,347$.

24. J. Balamurugan, S. M. Senthil Kumar, R. Thangamuthu, and A. Pandurangan, J. Mol. Catal. A: Chem., 2013, 372, 13.

25. L. Lin, J. Chen, H. Yao, Y. Chen, Y. Zheng, and X. Lin, Bioelectrochemistry, 2008, 73, 11.

26. P. C. Nien, P. Y. Chen, and K. C. Ho, Sens. Actuators, B, 2009, 140, 58.

27. K. Takahashi and T. Imato, Anal. Sci., 1999, 15, 181.

28. W. Zhang, R. Yuan, Y. Q. Chai, Y. Zhang, and S. H. Chen, Sens. Actuators, B, 2012, $166-167,601$.

29. A. Afkhami, F. Soltani-Felehgari, and T. Madrakian, Sens. Actuators, B, 2014, 196, 467.

30. J. Mathiyarasu, S. Senthilkumar, K. L. N. Phani, and V. Yegnaraman, Mater. Lett., 2008, 62, 571.

31. C. J. Weng, Y. L. Chen, C. M. Chien, S. C. Hsu, Y. S. Jhuo, J. M. Yeh, and C. F. Dai, Electrochim. Acta, 2013, 95, 162.

32. N. F. Atta and M. F. El-Kady, Sens. Actuators, B, 2010, 145, 299.

33. S. Kim, Y. Kim, A. Yu, J. Lee, S. Lee, C. Lee, M. Kim, and Y. Lee, Sens. Actuators, B, 2014, 196, 480.

34. M. Oyama, X. Chen, and X. Chen, Anal. Sci., 2014, 30, 529.

35. A. A. Ensafi, M. Taei, and T. Khayamian, J. Electroanal. Chem., 2009, 633, 212.

36. Y. Liu, Z. Su, Y. Zhang, L. Chen, T. Gu, S. Huang, Y. Liu, L. Sun, Q. Xie, and S. Yao, J. Electroanal. Chem., 2013 709, 19.

37. Y. Chen, W. Pei, S. Chen, X. Wu, S. Zhao, H. Wang, and H. Chen, Sens. Actuators, B, 2013, 188, 747.

38. S. Radhakrishnan, C. Sumathi, A. Umar, S. Jae Kim, J. Wilson, and V. Dharuman, Biosens. Bioelectron., 2013, 47, 133.

39. A. Et Taouil, F. Lallemand, J. Y. Hihn, J. M. Melot, V. Blondeau-Patissier, and B. Lakard, Ultrason. Sonochem., 2011, 18, 140.

40. A. Bello, M. Giannetto, G. Mori, R. Seeber, F. Terzi, and C. Zanardi, Sens. Actuators, B, 2007, 121, 430. 
41. Y. Chen, W. Pei, S. Chen, X. Wu, S. Zhao, and H. Wang, Sens. Actuators, B, 2013, 188, 747.

42. W. Si, W. Lei, Z. Han, Y. Zhang, Q. Hao, and M. Xia, Sens. Actuators, B, 2014, 193, 823.

43. G. Xu, B. Li, X. T. Cui, L. Ling, and X. Luo, Sens. Actuators, B, 2013, 188, 405.

44. K. C. Lin, T. H. Tsai, and S. M. Chen, Biosens. Bioelectron., 2010, 26, 608.

45. K. He, X. Wang, X. Meng, H. Zheng, and S. Suye, Sens. Actuators, B, 2014, 193, 212.

46. C. M. Long, M. A. Nascarella, and P. A. Valberg, Environ.
Pollut., 2013, 181, 271.

47. P. Takmakov, M. K. Zachek, R. B. Keithley, P. L. Walsh, C. Donley, G. S. Mccarty, and R. M. Wightman, Anal. Chem., 2010, 82, 2020.

48. K. E. Swider and D. R. Rolison, Langumir, 1999, 15, 3302.

49. N. F. Atta, I. Marawi, K. L. Petticrew, H. Zimmer, H. B. Mark Jr., and A. Galal, J. Electroanal. Chem., 1996, 408, 47.

50. M. J. Giz, B. Duong, and N. J. Tao, J. Electroanal. Chem., 1999, 465, 72 . 